Journal of Fluids Mechanics, September 2013, Vol. 730, pp. 220-224

Accepted for publication in J. Fluid Mech.

\title{
Two-scale dynamics of flow past a partial cross-stream array of tidal turbines
}

\author{
Takafumi Nishino $†$ and Richard H. J. Willden \\ Department of Engineering Science, University of Oxford, Parks Road, Oxford OX1 3PJ, UK
}

(Received 19 December 2012; revised 15 May 2013; accepted 25 June 2013)

The characteristics of flow past a partial cross-stream array of (idealised) tidal turbines are investigated both analytically and computationally to understand the mechanisms that determine the limiting performance of partial tidal fences. A two-scale analytical partial tidal fence model reported earlier is further extended by better accounting for the effect of array-scale flow expansion on device-scale dynamics, so that the new model is applicable to short fences (consisting of a small number of devices) as well as to long fences. The new model explains theoretically general trends of the limiting performance of partial tidal fences. The new model is then compared to three-dimensional Reynoldsaveraged Navier-Stokes (RANS) computations of flow past an array of various numbers (up to 40) of actuator disks. On the whole, the analytical model agrees well with the RANS computations, suggesting that the two-scale dynamics described in the analytical model predominantly determines the fence performance in the RANS computations as well. The comparison also suggests that the limiting performance of short partial fences depends on how much of device far-wake mixing takes place within the array near-wake region. This factor, however, depends on the structures of the wake and therefore on the type/design of devices to be arrayed.

Key words: channel flow, coastal engineering, shallow water flows

\section{Introduction}

Tidal stream/current power generation is one of the emerging technologies in the field of renewable energy. Various types of tidal stream devices (the majority can be described as underwater versions of wind turbines) are currently proposed and tested; however, it is generally recognised that a considerably large number of devices would be required to make a meaningful contribution to the future energy supply (regardless of the exact design of devices to be employed). The challenge here is how to design efficient tidal power farms, i.e., how to array such a large number of devices to maximise their overall power generation whilst keeping their impact on the natural environment to an acceptable level. The so-called 'tidal fences' (spanwise array of tidal stream devices) are promising on their own and also as a component of large tidal farms to be deployed in the future.

A series of important theories on the efficiency of tidal fences has been derived during the last several years. Garrett \& Cummins (2007) have reported an extension of the Lanchester-Betz theory (on the efficiency of wind power generation based on the balances of mass, momentum and energy). An important finding was the significance of the effect of tidal channel blockage; the hydrodynamic limit of power extraction by devices placed in a tidal channel is proportional to $(1-B)^{-2}$, where $B$ is the channel blockage (ratio

$\dagger$ Email address for correspondence: takafumi.nishino@eng.ox.ac.uk 
of the frontal-projected area of devices to the channel cross-sectional area). Houlsby et al. (2008) and Whelan et al. (2009) further extended this theory, explaining that an 'effective' channel blockage may change due to the free-surface effect (the effect of changes in water depth that accompany power extraction) and hence the limit of power extraction may also change, depending on the Froude number of the flow. It should be noted here that the above theories by Garrett \& Cummins (2007), Houlsby et al. (2008) and Whelan et al. (2009) are all concerned with the local efficiency of tidal devices; in other words, these theories assume that the installation of devices into a tidal channel does not change the amount of flow coming into the channel. In reality, however, the channel inflow may reduce unless the hydrodynamic drag caused by the installation of devices is negligibly small compared to other flow resistances through the channel, e.g., sea-bed friction (Garrett \& Cummins 2005). Vennell (2010, 2011) combined a simplified model of this effect (namely a channel dynamics model) with the local power extraction theory of Garrett \& Cummins (2007) to estimate the efficiency of a number of devices arrayed across the cross-sections of various types of tidal channels. Also, Vennell (2012) included the effect of drag acting on device supporting structures in his model.

A drawback to all theories/models described above (regardless of whether the channel dynamics effects are considered or not) is that they are not directly applicable to devices arrayed across only a part of the channel cross-section. They (explicitly or implicitly) assume that devices are regularly arrayed across the entire channel cross-section, i.e., they are concerned with 'full' (rather than 'partial') tidal fences. As will become clearer later in this paper, this limitation essentially comes from the assumption that the mixing of flow behind devices takes place only in a single 'far-wake' region; in this sense, all theories/models mentioned above can be described as being based on a single-scale wake mixing assumption. Two major examples that highlight the significance of considering 'partial' tidal fences are: (i) channels where a considerable portion of their cross-section needs to be unblocked in order to allow for navigation of vessels and so forth, and (ii) headland sites, where the channel cross-section is semi-infinitely wide (and may also be deep) and therefore cannot be fully blocked by the fence. In recognition of this, Nishino \& Willden $(2012 b)$ have derived another important extension of the Betz theory to explore the efficiency of a partial tidal fence by introducing an idea of scale separation between the flow around each device and that around the array. One of the key findings from the new model was the existence of optimal intra-device spacing to maximize the efficiency of a partial tidal fence; for an infinitely wide channel, for example, the limit of energy extraction significantly increases from the Betz limit of $59.3 \%$ (of the kinetic energy of undisturbed incoming flow) to another limit of $79.8 \%$ if the spacing is optimised. The new model accounts for the effects of device- and array-scale far-wake mixing separately and may therefore be referred to as the first (and presumably the simplest) tidal stream power generation model employing a multi-scale wake mixing concept.

In this paper we present an extension of the partial fence model of Nishino \& Willden $(2012 b)$ (hereafter NW12) to further investigate the mechanics of flow past a partial tidal fence. Also reported will be three-dimensional Reynolds-averaged Navier-Stokes (RANS) computations of an array of various numbers (up to 40) of actuator disks to support the investigation as well as to validate the analytical model. The NW12 model assumes that the device-scale wake mixing takes place much faster than the array-scale flow expansion (the two scales are completely separated). This assumption, however, is valid only when the number of devices is sufficiently large. In this new study we account for the effect of array-scale flow expansion on device-scale mass, momentum and energy balances, so that the extended model will be applicable to a short fence (consisting of a small number of devices) as well as to a long fence. As with the NW12 model the mass flow through the 
channel is assumed to be constant, i.e., we do not consider channel dynamics effects in this study. This is because our focus here is on the partial fence flow characteristics that are independent of the channel dynamics; a combined channel dynamics and partial fence model will be reported separately by Willden and Nishino (to be submitted) to discuss the effect of channel mass flow reduction. Assuming a constant mass flow means that the power extracted by the fence is much smaller than the 'potential' of the channel discussed by Garrett \& Cummins (2005) (or more generally, the drag caused by the installation of the fence is small enough not to change the balance of flow resistance inside and outside the entire channel, if we consider that the channel is a part of a large flow system). See e.g. Vennell (2013) for recent discussion on the efficiency of tidal fences that are large enough to cause reduction of the entire channel flow. Also neglected in this study are the free-surface effects, which have recently been examined by Vogel et al. (to be submitted) by extending the NW12 model.

It should be noted that both NW12 model and the new model are essentially based on 'one-dimensional' flow analysis. This means that these models neglect the non-uniformity of flow across each device area (for the device-scale problem) and that across the fence area (for the array-scale problem). For the device-scale problem, this assumption seems reasonable; the non-uniformity does not affect the (device-area-averaged) thrust or power significantly (Nishino \& Willden 2012a). For the array-scale problem, again this assumption seems reasonable if we consider that all turbines in the array are under the same operating conditions (constant torque, for example) and subject to a uniform inflow. As will be presented later, RANS simulations of a spanwise array of actuator disks show that the flow through the disks is nearly uniform across most of the array span (provided that all disks have the same flow resistance). This suggests that one-dimensional analysis is suitable for this partial tidal fence problem.

\section{Analytical model}

Figure 1 shows a schematic of the extended partial tidal fence model. We consider a finite number $(n)$ of devices arrayed across a part of the cross-section of a wide channel (note that $n=5$ in figure 1). Similarly to the NW12 model, three different blockages are defined, namely the global, local and array blockages. Here we define that the frontalprojected area of each device is $A_{D}$ and the cross-sectional area of the channel is $A_{C}$; hence the global blockage $B_{G}=n A_{D} / A_{C}$. We also define that the cross-sectional area of 'local flow passage' for each device (at the device location) is $A_{L}$ (which increases as the spacing between each device increases); hence the local blockage $B_{L}=A_{D} / A_{L}$. Finally, the entire array of devices can be considered (in the array-scale problem to be described later) as a single power-extracting fence of area $A_{A}=n A_{L}$; hence the array blockage $B_{A}=n A_{L} / A_{C}$. It should be noted that $B_{G}=B_{L} B_{A}$, i.e. only two of the three blockages are independent. To determine the full configuration of an array, we need to specify four parameters: $A_{C}, A_{D}, A_{L}$ and $n$; however, the relationships among these four parameters are described by any two of the three blockage ratios. See NW12 for further descriptions of the global, local and array blockages.

Following NW12 we will consider the balances of mass, momentum and energy in two different scales, namely the device and array scales. The difference from NW12, however, is that the present model accounts for the interaction of device- and array-scale problems a little further. The NW12 model was strictly only applicable to a 'long' partial fence consisting of a large number of devices since the model was based on the less general assumption that the two scales were completely separated (and it remained undefined how large the number of devices should be for this assumption to be acceptable). In the 


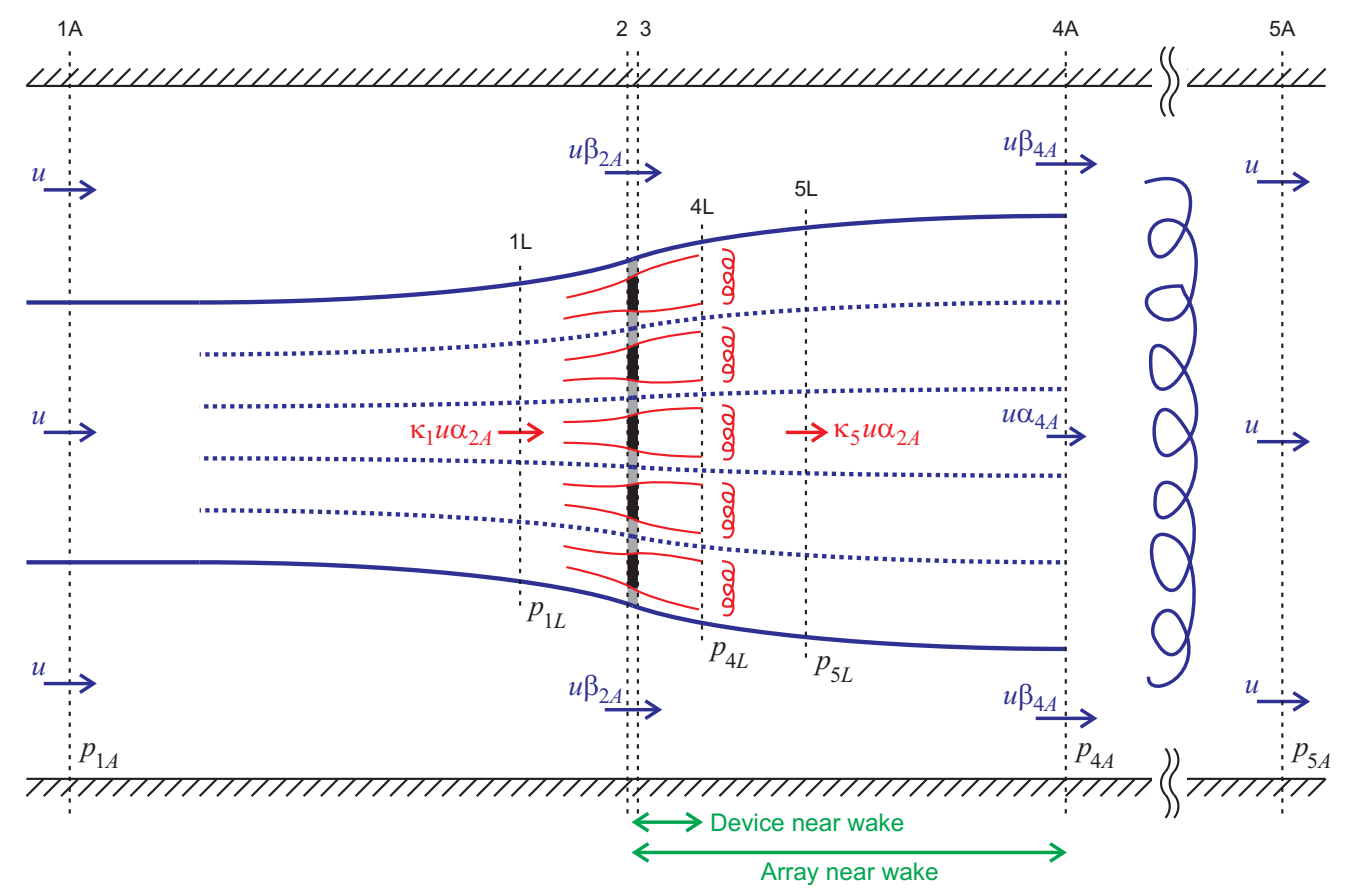

Figure 1. Schematic of the new partial tidal fence model.

present model, we relax this assumption by better accounting for the effects of arrayscale flow expansion on device-scale mass, momentum and energy balances. Hence the present model is more generic and provides a new theoretical framework to investigate the effect of the number of devices in the fence. As shown in figure 1 (and also in figure 2 , which describes the device-scale problem in more detail), we consider an array-scale stream-tube (dividing the channel flow into two flow passages, namely the array-scale bypass and core flow passages) and device-scale stream-tubes (dividing each local flow passage into the device-scale bypass and core flow passages); then define the following 8 streamwise locations:

- 1A: (array-scale) far upstream, where pressure and velocity are uniform across the channel cross-section;

- 1L: (device-scale) far upstream, where pressure and velocity are uniform across the cross-section of each local flow passage;

- 2: immediately upstream of the array/devices;

- 3: immediately downstream of the array/devices;

- 4L: (device-scale) pressure equilibrium location, where pressure equilibrates between the device-scale bypass and core flow passages;

- 5L: (device-scale) far downstream, where not only pressure but also velocity returns to be uniform across the cross-section of each local flow passage (due to device-scale wake mixing);

- 4A: (array-scale) pressure equilibrium location, where pressure equilibrates between the array-scale bypass and core flow passages; and

- 5A: (array-scale) far downstream, where not only pressure but also velocity returns to be uniform across the channel cross-section (due to array-scale wake mixing).

Stations $1 \mathrm{~L}, 2,3,4 \mathrm{~L}$ and $5 \mathrm{~L}$ are employed in the device-scale problem, whereas $1 \mathrm{~A}$, 


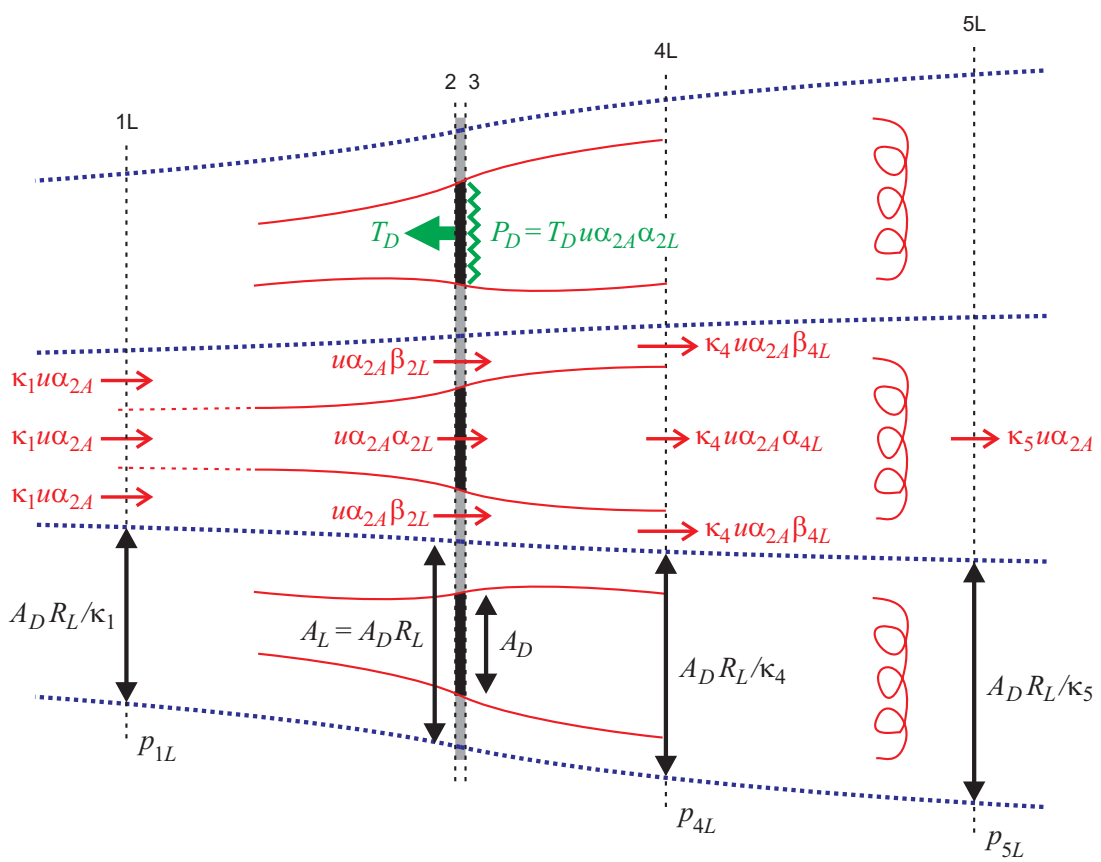

FiguRE 2. Enlarged schematic of the model; note that $R_{L}=1 / B_{L}$ and $\kappa_{i}=1 / \lambda_{i}$.

2, 3, 4A and 5A are used in the array-scale problem. As will be described later one by one, velocities for the core and bypass flow passages (at each station and for each scale problem) are described using coefficients $\alpha$ and $\beta$, which essentially represent the ratios of the core $(\alpha)$ and bypass $(\beta)$ flow velocities to the 'reference' velocity for each scale problem ( $u$ for the array-scale problem and $u \alpha_{2 A}$ for the device-scale problem). Also, pressures at stations $1 \mathrm{~A}, 4 \mathrm{~A}, 5 \mathrm{~A}, 1 \mathrm{~L}, 4 \mathrm{~L}$ and $5 \mathrm{~L}$ are described as $p_{1 A}, p_{4 A}, p_{5 A}, p_{1 L}, p_{4 L}$ and $p_{5 L}$, respectively.

It should be noted that the wake mixing is assumed to take place only in the device far-wake region (between stations $4 \mathrm{~L}$ and $5 \mathrm{~L}$ ) and array far-wake region (between stations $4 \mathrm{~A}$ and $5 \mathrm{~A}$ ). Also, the present model assumes that station $5 \mathrm{~L}$ is somewhere upstream of station 4A, i.e., the device-scale wake mixing is completed in the array near-wake region (thus the array-scale core flow velocity is uniform across the array-scale core flow passage at station $4 \mathrm{~A}$ ). This assumption may not fully hold when the number of devices $(n)$ is small. Nevertheless, the present model agrees fairly well with the three-dimensional RANS actuator disk computations, as will be shown later in Section 3. Further details of the model are described below.

\subsection{Array-scale mass conservation}

First, we consider the conservation of mass flow for the array-scale bypass and core flow passages. As described in figure $1, u$ denotes the uniform velocity far upstream (station 1A) and far downstream (station $5 \mathrm{~A}$ ), $u \beta_{2 A}$ and $u \beta_{4 A}$ the bypass flow velocities at the array location and at station $4 \mathrm{~A}$, respectively, and $u \alpha_{4 A}$ the core flow velocity at station $4 \mathrm{~A}$. We also define the cross-sectional average of core flow velocity at the array location as $u \alpha_{2 A}$. Since the (nominal) 'array area' is $A_{A}\left(=n A_{L}=n A_{D} R_{L}\right.$, where $\left.R_{L}=1 / B_{L}\right)$, the core flow cross-sectional areas at stations $1 \mathrm{~A}$ and $4 \mathrm{~A}$ are $A_{A} \alpha_{2 A}$ and $A_{A} \alpha_{2 A} / \alpha_{4 A}$, respectively, due to the conservation of mass. Also, since the channel cross-sectional area 
$A_{C}=A_{A} R_{A}\left(=n A_{D} R_{L} R_{A}\right.$, where $\left.R_{A}=1 / B_{A}\right)$, the bypass flow cross-sectional areas at stations $1 \mathrm{~A}$ and $4 \mathrm{~A}$ are $A_{A}\left(R_{A}-\alpha_{2 A}\right)$ and $A_{A}\left(R_{A}-\alpha_{2 A} / \alpha_{4 A}\right)$, respectively.

It is also obtained, again from the conservation of mass, that

$$
\beta_{2 A}=\frac{R_{A}-\alpha_{2 A}}{R_{A}-1}
$$

and

$$
\beta_{4 A}=\frac{R_{A}-\alpha_{2 A}}{R_{A}-\alpha_{2 A} / \alpha_{4 A}} .
$$

It should be noted that $\alpha_{4 A}$ is also a function of $\alpha_{2 A}$ (and vice versa); the relationship between the two is obtained by considering the momentum balance, as will be described later in Section 2.7. Also note that $\beta_{2 A}$ described above is just for completeness of the description of the flow system and is not required to solve the model.

\subsection{Array-scale flow expansion factors}

Next, we attempt to express the array-scale flow expansion between stations $1 \mathrm{~L}$ and $5 \mathrm{~L}$ systematically so that it can be included in the device-scale problem. In NW12 the crosssectional area of each local flow passage was constant throughout the passage $\left(A_{L}=\right.$ $\left.A_{D} R_{L}\right)$ as we assumed that all device-scale flow events take place much faster than the array-scale flow expansion. In the present model, each local flow passage expands toward downstream (i.e. the local cross-sectional area is smaller than $A_{D} R_{L}$ upstream and larger than $A_{D} R_{L}$ downstream of the device location) due to the array-scale flow expansion. As shown in figure 2, here we introduce array-scale flow expansion factors, $\lambda_{1}, \lambda_{4}$ and $\lambda_{5}$ (and their inverses, $\kappa_{1}=1 / \lambda_{1}, \kappa_{4}=1 / \lambda_{4}$ and $\kappa_{5}=1 / \lambda_{5}$ ) for stations $1 \mathrm{~L}, 4 \mathrm{~L}$ and $5 \mathrm{~L}$, respectively, so that the local cross-sectional areas are expressed as $\lambda_{1} A_{D} R_{L}, \lambda_{4} A_{D} R_{L}$ and $\lambda_{5} A_{D} R_{L}$ for the three stations. We assume that these flow expansion factors do not change for all $n$ local flow passages, i.e. all local passages expand equivalently. As will become clear later, $\lambda_{1}$ and $\lambda_{4}$ (or alternatively, $\kappa_{1}$ and $\kappa_{4}$ ) need to be specified in order to solve the device-scale problem.

To specify $\lambda_{1}$ and $\lambda_{4}$ the following should be taken into account. For $\lambda_{1}$ the maximum possible value is 1 , which is the case when the number of devices $(n)$ is so large that the distance between stations $1 \mathrm{~L}$ and 2 becomes negligibly small compared to the distance between stations $1 \mathrm{~A}$ and 2 (i.e. the situation considered in NW12), whereas the minimum possible value (by definition) is $\alpha_{2 A}$, which is the case when $n=1$ and thus station $1 \mathrm{~L}$ becomes identical to station $1 \mathrm{~A}$ (note that the local cross-sectional area at station $1 \mathrm{~A}$ is $A_{A} \alpha_{2 A} / n=A_{D} R_{L} \alpha_{2 A}$ ). Similarly, for $\lambda_{4}$ the minimum possible value is 1 (for $n \rightarrow \infty$ ) whereas the maximum possible value (by definition) is $\alpha_{2 A} / \alpha_{4 A}$ (for $n=1$; note that the local cross-sectional area at station $4 \mathrm{~A}$ is $\left.A_{D} R_{L} \alpha_{2 A} / \alpha_{4 A}\right)$.

In this study we use simple power-law approximations to model the dependence of $\lambda_{1}$ and $\lambda_{4}$ on $1 / n$, thereby specify $\lambda_{1}$ and $\lambda_{4}$ as follows:

$$
\lambda_{1}=\frac{1}{\kappa_{1}}=1+\left(\frac{1}{n}\right)^{\gamma_{1}}\left(\alpha_{2 A}-1\right)
$$

and

$$
\lambda_{4}=\frac{1}{\kappa_{4}}=1+\left(\frac{1}{n}\right)^{\gamma_{4}}\left(\frac{\alpha_{2 A}}{\alpha_{4 A}}-1\right) .
$$

where $\gamma_{1}$ and $\gamma_{4}$ are the scaling exponents that determine the dependence of $\lambda_{1}$ and $\lambda_{4}$ on $1 / n$. Unless specified, $\gamma_{1}=\gamma_{4}=1$ is used in this study as a first order approximation. 
As will be discussed later in Section 4, more complex model functions of $\lambda_{1}$ and $\lambda_{4}$ may also be devised and used in future studies.

\subsection{Device-scale mass conservation}

As the array-scale flow expansion factors have been introduced, now we can consider the conservation of mass flow for the device-scale problem. Since the mean velocity (for each local flow passage) at the array location is $u \alpha_{2 A}$, the velocities at stations $1 \mathrm{~L}$ and $5 \mathrm{~L}$ are $\kappa_{1} u \alpha_{2 A}$ and $\kappa_{5} u \alpha_{2 A}$, respectively, and also the mean velocity at station $4 \mathrm{~L}$ is $\kappa_{4} u \alpha_{2 A}$ due to the conservation of mass for each local flow passage.

As shown in figure 2, we express the local bypass flow velocities at stations 2 and $4 \mathrm{~L}$ as $u \alpha_{2 A} \beta_{2 L}$ and $\kappa_{4} u \alpha_{2 A} \beta_{4 L}$. Also, we express the local core flow velocities at stations 2 and $4 \mathrm{~L}$ as $u \alpha_{2 A} \alpha_{2 L}$ and $\kappa_{4} u \alpha_{2 A} \alpha_{4 L}$. Due to the conservation of mass for the local core flow, the local core flow areas at stations $1 \mathrm{~L}$ and $4 \mathrm{~L}$ are $A_{D} \alpha_{2 L} / \kappa_{1}$ and $A_{D} \alpha_{2 L} / \alpha_{4 L} \kappa_{4}$; therefore the local bypass flow areas at stations $1 \mathrm{~L}$ and $4 \mathrm{~L}$ are $A_{D}\left(R_{L}-\alpha_{2 L}\right) / \kappa_{1}$ and $A_{D}\left(R_{L}-\alpha_{2 L} / \alpha_{4 L}\right) / \kappa_{4}$, respectively.

It is also obtained from the conservation of mass for the local bypass flow that

$$
\beta_{2 L}=\frac{R_{L}-\alpha_{2 L}}{R_{L}-1}
$$

and

$$
\beta_{4 L}=\frac{R_{L}-\alpha_{2 L}}{R_{L}-\alpha_{2 L} / \alpha_{4 L}} .
$$

It should be noted that $\alpha_{4 L}$ is also a function of $\alpha_{2 L}$ (and vice versa); the relationship between the two is obtained by considering the momentum balance, as will be described later in Section 2.7. Also note that $\beta_{2 L}$ described above is just for completeness of the description of the flow system and is not required to solve the model.

\subsection{Device-scale energy balance}

Now we derive the device-scale energy balance equations. First, we consider each local bypass flow passage between stations $1 \mathrm{~L}$ and $4 \mathrm{~L}$ as a single 'control-volume', for which the energy equation can be described as

$$
\begin{aligned}
& \left(\frac{1}{2} \rho\left(\kappa_{1} u \alpha_{2 A}\right)^{2}+p_{1 L}\right) u \alpha_{2 A} A_{D}\left(R_{L}-\alpha_{2 L}\right) \\
& =\left(\frac{1}{2} \rho\left(\kappa_{4} u \alpha_{2 A} \beta_{4 L}\right)^{2}+p_{4 L}\right) u \alpha_{2 A} \beta_{4 L} A_{D}\left(R_{L}-\frac{\alpha_{2 L}}{\alpha_{4 L}}\right),
\end{aligned}
$$

where $\rho$ is the density of fluid and $p_{1 L}$ and $p_{4 L}$ are the pressures at stations $1 \mathrm{~L}$ and $4 \mathrm{~L}$. By substituting (2.6) the above equation can be simplified to

$$
\frac{1}{2} \rho\left(u \alpha_{2 A}\right)^{2}\left(\kappa_{1}^{2}-\kappa_{4}^{2} \beta_{4 L}^{2}\right)+p_{1 L}-p_{4 L}=0 .
$$

Note that this equation (2.8) can also be seen as a Bernoulli equation for a stream-line along the local bypass flow passage.

Next, we consider each local core flow passage between stations $1 \mathrm{~L}$ and $4 \mathrm{~L}$ as a single control-volume, for which the energy equation can be described as

$$
\begin{aligned}
& \left(\frac{1}{2} \rho\left(\kappa_{1} u \alpha_{2 A}\right)^{2}+p_{1 L}\right) u \alpha_{2 A} \alpha_{2 L} A_{D} \\
& \quad=\left(\frac{1}{2} \rho\left(\kappa_{4} u \alpha_{2 A} \alpha_{4 L}\right)^{2}+p_{4 L}\right) u \alpha_{2 A} \alpha_{2 L} A_{D}+P_{D},
\end{aligned}
$$


where $P_{D}=u \alpha_{2 A} \alpha_{2 L} T_{D}$ is the power removed by the device (at the device location) and $T_{D}$ the thrust on the device. Hence the above equation can be simplified to

$$
\frac{1}{2} \rho\left(u \alpha_{2 A}\right)^{2}\left(\kappa_{1}^{2}-\kappa_{4}^{2} \alpha_{4 L}^{2}\right)+p_{1 L}-p_{4 L}=\frac{T_{D}}{A_{D}} .
$$

Again, this equation (2.10) can be seen as a Bernoulli equation for a stream-line along the local core flow passage.

\subsection{Array-scale energy balance}

Next, we derive the array-scale energy balance equations. The energy equation for the array-scale bypass flow passage between stations $1 \mathrm{~A}$ and $4 \mathrm{~A}$ can be described as

$$
\begin{aligned}
& \left(\frac{1}{2} \rho u^{2}+p_{1 A}\right) u A_{A}\left(R_{A}-\alpha_{2 A}\right) \\
& =\left(\frac{1}{2} \rho\left(u \beta_{4 A}\right)^{2}+p_{4 A}\right) u \beta_{4 A} A_{A}\left(R_{A}-\frac{\alpha_{2 A}}{\alpha_{4 A}}\right),
\end{aligned}
$$

where $p_{1 A}$ and $p_{4 A}$ are the pressures at stations $1 \mathrm{~A}$ and $4 \mathrm{~A}$. By substituting (2.2) the above equation can be simplified to

$$
\frac{1}{2} \rho u^{2}\left(1-\beta_{4 A}^{2}\right)+p_{1 A}-p_{4 A}=0 .
$$

Meanwhile, the energy equation for the array-scale core flow passage between stations $1 \mathrm{~A}$ and $4 \mathrm{~A}$ can be described as

$$
\left(\frac{1}{2} \rho u^{2}+p_{1 A}\right) u \alpha_{2 A} A_{A}=\left(\frac{1}{2} \rho\left(u \alpha_{4 A}\right)^{2}+p_{4 A}\right) u \alpha_{2 A} A_{A}+P_{A}
$$

where $P_{A}$ is the power removed by the array as a single power-extracting fence. In other words, $P_{A}$ represents not only the power removed by the devices at the device location but also the power lost (to heat) due to the device-scale wake mixing. Since the crosssectionally averaged velocity across this single power extracting fence is $u \alpha_{2 A}, P_{A}$ can be described as $P_{A}=u \alpha_{2 A} T_{A}$, where $T_{A}=n T_{D}$ is the thrust on the entire array (note that $P_{D}=u \alpha_{2 A} \alpha_{2 L} T_{D}$; therefore $P_{A}=n P_{D} / \alpha_{2 L}$ ). Hence (2.13) can be simplified to

$$
\frac{1}{2} \rho u^{2}\left(1-\alpha_{4 A}^{2}\right)+p_{1 A}-p_{4 A}=\frac{T_{A}}{A_{A}} .
$$

\subsection{Thrust and power coefficients}

Similarly to NW12, here we define three different thrust coefficients, namely local $\left(C_{T L}\right)$, array $\left(C_{T A}\right)$ and global $\left(C_{T G}\right)$ thrust coefficients:

$$
\begin{aligned}
C_{T L} & =\frac{T_{D}}{\frac{1}{2} \rho\left(u \alpha_{2 A}\right)^{2} A_{D}}=\kappa_{4}^{2}\left(\beta_{4 L}^{2}-\alpha_{4 L}^{2}\right) ; \\
C_{T A} & =\frac{T_{A}}{\frac{1}{2} \rho u^{2} A_{A}}=\left(\beta_{4 A}^{2}-\alpha_{4 A}^{2}\right) ; \\
C_{T G} & =\frac{n T_{D}}{\frac{1}{2} \rho u^{2} n A_{D}}=\alpha_{2 A}^{2} C_{T L} .
\end{aligned}
$$

Note that (2.15) is derived from the device-scale energy balances (2.8) and (2.10), whilst (2.16) is derived from the array-scale energy balances (2.12) and (2.14). Of importance is that the device- and array-scale problems are linked to each other by $T_{A}=n T_{D}$ and 
$A_{A}=n A_{D} / B_{L}$. This means that $C_{T L}$ obtained from (2.15) and $C_{T A}$ obtained from (2.16) must satisfy the following relationship:

$$
C_{T A}=\alpha_{2 A}^{2} B_{L} C_{T L} .
$$

We also define three different power coefficients, namely local $\left(C_{P L}\right)$, array $\left(C_{P A}\right)$ and global $\left(C_{P G}\right)$ power coefficients:

$$
\begin{aligned}
C_{P L} & =\frac{P_{D}}{\frac{1}{2} \rho\left(u \alpha_{2 A}\right)^{3} A_{D}}=\alpha_{2 L} \kappa_{4}^{2}\left(\beta_{4 L}^{2}-\alpha_{4 L}^{2}\right) ; \\
C_{P A} & =\frac{P_{A}}{\frac{1}{2} \rho u^{3} A_{A}}=\alpha_{2 A}\left(\beta_{4 A}^{2}-\alpha_{4 A}^{2}\right) ; \\
C_{P G} & =\frac{n P_{D}}{\frac{1}{2} \rho u^{3} n A_{D}}=\alpha_{2 A}^{3} C_{P L} .
\end{aligned}
$$

It should be reminded that one of our primary objectives in this study is to examine how the number of devices forming a partial tidal fence affects its optimal local blockage (for a given global blockage) to maximise $C_{P G}$, which represents the power removed by the devices at the device location, $n P_{D}$, relative to the 'available' power, i.e. the power of undisturbed flow passing through the total device area. The power removed at the device location, $n P_{D}$ (or alternatively its normalised coefficient, $C_{P G}$ ) can be referred to as the hydrodynamic limit of power extraction (for given conditions), in the sense that, in reality, only a part of this power can be extracted as useful power and the rest will be wasted either (i) by generating heat immediately at the device location due to friction, mechanical loss, etc.; or (ii) by generating non-streamwise and/or time-dependent fluid motion, such as mean swirling, large-scale coherent structures and turbulence, the power of which is also eventually turned into heat due to (device-scale) wake mixing.

\subsection{Momentum balance}

So far we have derived (from the balances of mass and energy) that all thrust and power coefficients are determined by the combination of $\alpha_{2 A}, \alpha_{4 A}, \alpha_{2 L}$ and $\alpha_{4 L}$ (and also the number of devices, $n$, to determine $\kappa_{4}$ in the present model, cf. (2.4)). As already noted, the relationship between $\alpha_{2 A}$ and $\alpha_{4 A}$ in the array-scale problem and that between $\alpha_{2 L}$ and $\alpha_{4 L}$ in the device-scale problem are obtained by considering the momentum balance in each problem, which will be described below.

First, we consider the entire channel between stations $1 \mathrm{~A}$ and $4 \mathrm{~A}$ as a single control volume, for which the momentum balance equation can be described as

$$
\begin{aligned}
& p_{1 A} A_{A} R_{A}- p_{4 A} A_{A} R_{A}-T_{A}=\rho\left(u \alpha_{4 A}\right)^{2} \frac{A_{A} \alpha_{2 A}}{\alpha_{4 A}}-\rho u^{2} A_{A} \alpha_{2 A} \\
&+\rho\left(u \beta_{4 A}\right)^{2} A_{A}\left(R_{A}-\frac{\alpha_{2 A}}{\alpha_{4 A}}\right)-\rho u^{2} A_{A}\left(R_{A}-\alpha_{2 A}\right),
\end{aligned}
$$

which can be simplified to

$$
\frac{\left(p_{1 A}-p_{4 A}\right) R_{A}}{\frac{1}{2} \rho u^{2}}-C_{T A}=2 \alpha_{2 A}\left(\alpha_{4 A}-1\right)+2\left(R_{A}-\alpha_{2 A}\right)\left(\beta_{4 A}-1\right) .
$$

Note that the left-hand side of (2.23) represents the (non-dimensionalised) force acting on the channel between stations $1 \mathrm{~A}$ and $4 \mathrm{~A}$, whereas the right-hand side represents the corresponding momentum change through this control volume. By substituting (2.12) and (2.16) to remove $p_{1 A}-p_{4 A}$ and $C_{T A}$ from (2.23), we obtain the following equation 
to be satisfied:

$$
R_{A}\left(\beta_{4 A}^{2}-1\right)-\left(\beta_{4 A}^{2}-\alpha_{4 A}^{2}\right)=2 \alpha_{2 A}\left(\alpha_{4 A}-1\right)+2\left(R_{A}-\alpha_{2 A}\right)\left(\beta_{4 A}-1\right)
$$

Since $\beta_{4 A}$ is a function of $\alpha_{2 A}, \alpha_{4 A}$ and $R_{A}$ as given by (2.2), the above equation (2.24) provides the relationship between $\alpha_{2 A}$ and $\alpha_{4 A}$ (for a given $R_{A}$ or $B_{A}$ ).

Next, we consider the momentum balance for each local flow passage between stations $1 \mathrm{~L}$ and $4 \mathrm{~L}$ to derive the relationship between $\alpha_{2 L}$ and $\alpha_{4 L}$. A difficulty here is that each local flow passage expands toward downstream (except for the case with $n \rightarrow \infty$ ); hence pressure along this expanding (non-parallel) stream-tube surface also affects the device-scale momentum balance. Using $p^{*} A^{*}$ to denote the (unknown) surface integral of the streamwise component of pressure force acting on this stream-tube, the momentum balance equation can be described as

$$
\begin{aligned}
p_{1 L} \frac{A_{D} R_{L}}{\kappa_{1}}+ & p^{*} A^{*}-p_{4 L} \frac{A_{D} R_{L}}{\kappa_{4}}-T_{D}=\rho\left(\kappa_{4} u \alpha_{2 A} \alpha_{4 L}\right)^{2} \frac{A_{D} \alpha_{2 L}}{\alpha_{4 L} \kappa_{4}}-\rho\left(\kappa_{1} u \alpha_{2 A}\right)^{2} \frac{A_{D} \alpha_{2 L}}{\kappa_{1}} \\
& +\rho\left(\kappa_{4} u \alpha_{2 A} \beta_{4 L}\right)^{2} \frac{A_{D}}{\kappa_{4}}\left(R_{L}-\frac{\alpha_{2 L}}{\alpha_{4 L}}\right)-\rho\left(\kappa_{1} u \alpha_{2 A}\right)^{2} \frac{A_{D}\left(R_{L}-\alpha_{2 L}\right)}{\kappa_{1}} .
\end{aligned}
$$

In order to close the model, here we consider the following approximation:

$$
p_{1 L} \frac{A_{D} R_{L}}{\kappa_{1}}+p^{*} A^{*} \approx p_{1 L} \frac{A_{D} R_{L}}{\kappa_{4}} .
$$

This physically means that the additional contribution from the expanding stream-tube surface, $p^{*} A^{*}$, is approximated by the additional force that would be exerted at station $1 \mathrm{~L}$ if the cross-sectional area at station $1 \mathrm{~L}\left(A_{D} R_{L} / \kappa_{1}\right)$ was increased to that at station $4 \mathrm{~L}\left(A_{D} R_{L} / \kappa_{4}\right)$. (This approximation is exact if the pressure along the stream-tube is constant at $p_{1 L}$; in reality, the pressure should be higher than $p_{1 L}$ upstream of the array and lower than $p_{1 L}$ downstream of the array. Our RANS computations suggest that this approximation tends to somewhat overpredict $p^{*} A^{*}$ as the effect of lower pressure in the downstream surpasses the effect of higher pressure in the upstream. Note, however, that the contribution of $p^{*} A^{*}$ to the momentum balance is minor in the first place unless the number of devices $n$ is very small and the difference of $\kappa_{1}$ and $\kappa_{4}$ is significant.) By using (2.26), (2.25) can be simplified to

$$
\frac{\left(p_{1 L}-p_{4 L}\right) R_{L}}{\frac{1}{2} \rho\left(u \alpha_{2 A}\right)^{2} \kappa_{4}}-C_{T L}=2 \alpha_{2 L}\left(\kappa_{4} \alpha_{4 L}-\kappa_{1}\right)+2\left(R_{L}-\alpha_{2 L}\right)\left(\kappa_{4} \beta_{4 L}-\kappa_{1}\right) .
$$

By substituting (2.8) and (2.15) to remove $p_{1 L}-p_{4 L}$ and $C_{T L}$, we obtain the following equation to be satisfied between $\alpha_{2 L}$ and $\alpha_{4 L}$ :

$$
\begin{aligned}
\frac{R_{L}}{\kappa_{4}}\left(\kappa_{4}^{2} \beta_{4 L}^{2}-\kappa_{1}^{2}\right) & -\kappa_{4}^{2}\left(\beta_{4 L}^{2}-\alpha_{4 L}^{2}\right) \\
& =2 \alpha_{2 L}\left(\kappa_{4} \alpha_{4 L}-\kappa_{1}\right)+2\left(R_{L}-\alpha_{2 L}\right)\left(\kappa_{4} \beta_{4 L}-\kappa_{1}\right) .
\end{aligned}
$$

In summary, (2.28) and (2.24) are the device- and array-scale momentum equations to be solved, respectively. For a given set of $n, R_{L}$ (or $B_{L}$ ) and $R_{A}$ (or $B_{A}$ ), we can obtain $\alpha_{4 L}$ as a function of $\alpha_{2 L}$ by solving (2.28) and thus $C_{T L}$ from (2.15), whilst we can also obtain $\alpha_{4 A}$ as a function of $\alpha_{2 A}$ by solving (2.24) and thus $C_{T A}$ from (2.16). Here $C_{T L}$ and $C_{T A}$ must satisfy (2.18) as already noted in Section 2.6. Since $C_{T A}$ increases whilst $C_{T L}$ decreases as $\alpha_{2 A}$ decreases from 1, the value of $\alpha_{2 A}$ that satisfies (2.18) is uniquely determined for a given set of $\alpha_{2 L}, R_{L}, R_{A}$ and $n$. Eventually, all thrust and power coefficients are uniquely determined for a given set of $\alpha_{2 L}, R_{L}, R_{A}$ and $n$. Note that the present model returns to the NW12 model when $n \rightarrow \infty\left(\kappa_{1}=\kappa_{4}=1\right)$. 


\subsection{Wake mixing loss factor}

Before presenting results of the new model, we consider another non-dimensional factor related to the efficiency of tidal fences, called 'wake mixing loss factor'. Here we express the total power removed from the entire channel flow between stations $1 \mathrm{~A}$ and $5 \mathrm{~A}$ as $P_{C}$. Since the energy equation for the entire channel between stations $1 \mathrm{~A}$ and $5 \mathrm{~A}$ can be described as

$$
\left(\frac{1}{2} \rho u^{2}+p_{1 A}\right) u A_{C}=\left(\frac{1}{2} \rho u^{2}+p_{5 A}\right) u A_{C}+P_{C}
$$

whereas the momentum equation can be described as

$$
p_{1 A} A_{C}-p_{5 A} A_{C}-n T_{D}=0
$$

the total power removed from the channel is $P_{C}=n T_{D} u$. Meanwhile, the power removed by the devices at the device location is $n P_{D}$ as already discussed in Section 2.6; therefore the power lost to heat due to (device- and array-scale) wake mixing is $P_{W}=P_{C}-n P_{D}$. The ratio of $n P_{D}$ to $P_{C}$ is sometimes referred to as 'basin efficiency' or 'farm efficiency' in order to distinguish it from the efficiency represented by the global power coefficient $C_{P G}$, although the same type of efficiency has also been introduced by Corten (2000) for wind turbines, to which the term 'basin' is not relevant. In this study, we define the ratio of $P_{W}$ to $P_{C}$ (rather than $n P_{D}$ to $P_{C}$ ) as 'wake mixing loss factor' of tidal fences:

$$
\text { wake mixing loss factor }=\frac{P_{W}}{P_{C}}=\frac{n T_{D} u-n P_{D}}{n T_{D} u}=1-\alpha_{2 A} \alpha_{2 L} \text {. }
$$

Note that $\alpha_{2 A} \alpha_{2 L}$ is the ratio of the velocity through each device to the velocity far upstream of the entire array, $u$ (cf. figures 1 and 2); hence the wake mixing loss factor defined above is identical to the global axial induction factor of each device:

$$
a_{G}=1-\frac{u \alpha_{2 A} \alpha_{2 L}}{u}=1-\alpha_{2 A} \alpha_{2 L} .
$$

\subsection{Results}

Below we present some examples of the solution of the new analytical model to examine differences in performance characteristics between short and long partial tidal fences.

First we consider a fixed global blockage $\left(B_{G}=0.001\right)$; hence here we have only three independent parameters: the number of devices $n$, local blockage $B_{L}\left(=1 / R_{L}\right)$, and one of the parameters that determine the operating condition of devices $\left(\alpha_{2 L}\right.$ for example). Figure 3 shows contours of the global power coefficient $C_{P G}$ plotted with respect to $B_{L}$ and the wake mixing loss factor $\left(1-\alpha_{2 A} \alpha_{2 L}\right)$ for $n=4$ and 16 (representing relatively short and long fences). Note that the wake mixing loss factor is identical to the global induction factor of each device; hence the loss factor $=0$ means that the devices do not decelerate the flow at all (therefore $C_{P G}=0$ ) whereas the loss factor $=1$ means that the devices completely block the flow (therefore again $C_{P G}=0$ ). For both short and long fence cases, $C_{P G}$ is maximised when the loss factor is around 0.33 to 0.45 (depending on $B_{L}$ as shown by the white lines in the figure). This means that we can increase the output power (for a given array configuration) by reducing the flow through each device by up to about 33 to $45 \%$ of the undisturbed channel flow, although the wake mixing loss also increases as the flow through each device is reduced (also note that these results are for arrays that do not cause reduction of channel inflow, as described earlier). Of further interest is the effect of $B_{L}$. For the longer fence, the effect is very similar to that predicted by NW12; $C_{P G}$ increases as $B_{L}$ increases up to about 0.3 to 0.4 and then decreases as 

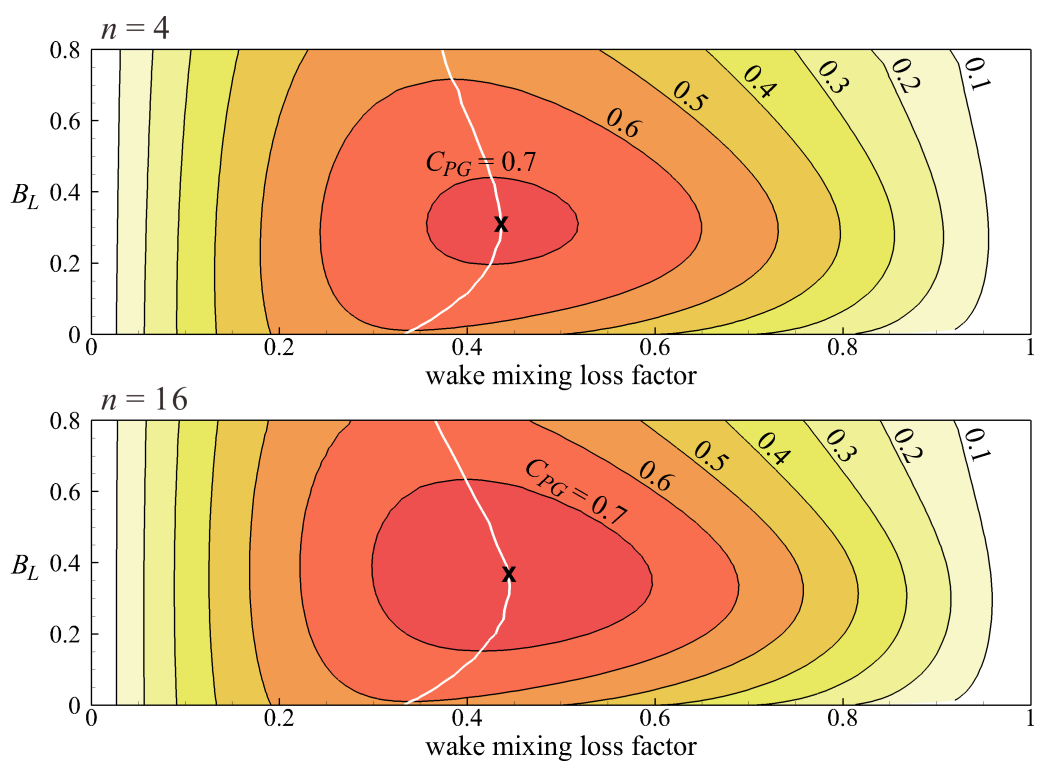

FiguRE 3. Relationship between global power coefficient $C_{P G}$ and wake mixing loss factor as a function of local blockage $B_{L}$, for two different numbers of devices: $n=4$ and 16. Global blockage $B_{G}$ is fixed at 0.001 . White lines represent $C_{P G \max }$ (the maximal $C_{P G}$ for given $B_{L}$ ) and 'x' indicates the peak of $C_{P G \max }$.

$B_{L}$ further increases. This means that, even without increasing the wake mixing loss, we can increase the power by optimising the array configuration. For the shorter fence, the overall trend is still similar but the decrease in $C_{P G}$ at higher $B_{L}$ is more significant and the optimal $B_{L}$ decreases compared to the longer fence case.

Figure 4 compares the effects of $B_{L}$ on the maximal $C_{P G}$ (hereafter $C_{P G \max }$ ) and also the values of $C_{P L}$ and $\alpha_{2 A}^{3}$ at the optimal operating condition (that yields $C_{P G \max }$ for given $B_{L}$ ) for $n=4,8,16$ and 100. Again the global blockage is fixed at $B_{G}=0.001$. As discussed in NW12, the optimal local blockage to maximise the limit of power extraction by a partial tidal fence is determined by the balance between the local blockage effect, represented by $C_{P L}$, and the array-scale choking (or flow reduction) effect, represented by $\alpha_{2 A}^{3}$. Note that $C_{P G}=\alpha_{2 A}^{3} C_{P L}$ as shown in (2.21). Of importance here is the effect of the number of devices, $n$, on the local power coefficient, $C_{P L}$. As can be seen from figure 4, the local blockage effect (represented by $C_{P L}$ ) diminishes as $n$ decreases; this is because the expansion of each local flow passage (due to the array-scale flow expansion, represented by $\kappa_{1}$ and $\kappa_{4}$ ) becomes more significant as the number of devices decreases. This results in the decrease in $C_{P G \max }$ and also alters the balance between $C_{P L}$ and $\alpha_{2 A}^{3}$ in such a way that the optimal $B_{L}$ decreases as the number of devices decreases.

The above effects of the number of devices are important not only for low global blockage cases but also for higher global blockage cases. Figure 5 shows contours of $C_{P G \max }$ plotted with respect to $B_{L}$ and $B_{G}$ for $n=4$ and 16 (again representing relatively short and long fences). As can be seen from the figure, the optimal $B_{L}$ (to maximise $C_{P G \max }$ ) is smaller for the shorter fence than for the longer fence, regardless of the global blockage $B_{G}$ (although the optimal $B_{L}$ for each fence does depend on $B_{G}$ ). Note that, for both short and long fence cases, we can increase the output power by optimising $B_{L}$ (or optimising the spacing between each device) even when $B_{G}$ is high; at $B_{G}=0.4$, for 

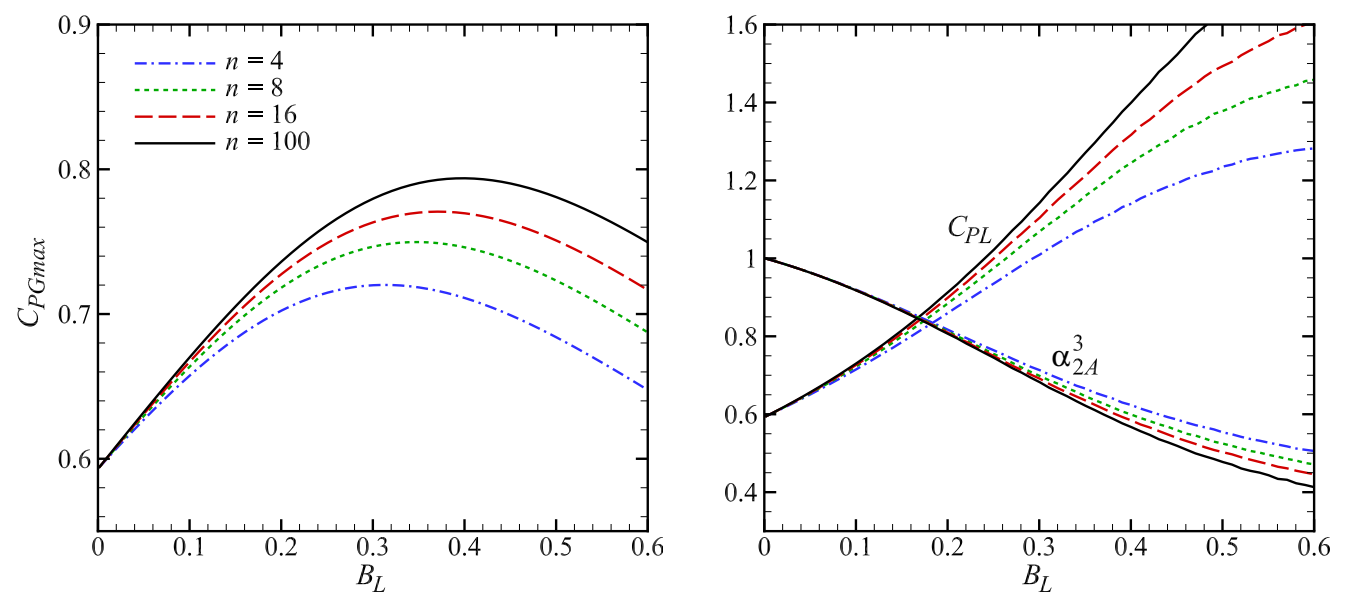

Figure 4. Effects of local blockage $B_{L}$ : (left) on $C_{P G \max }$ and (right) on the values of $C_{P L}$ and $\alpha_{2 A}^{3}$ that yield $C_{P G \max }$; for $n=4,8,16$ and 100 . Global blockage $B_{G}$ is fixed at 0.001 .
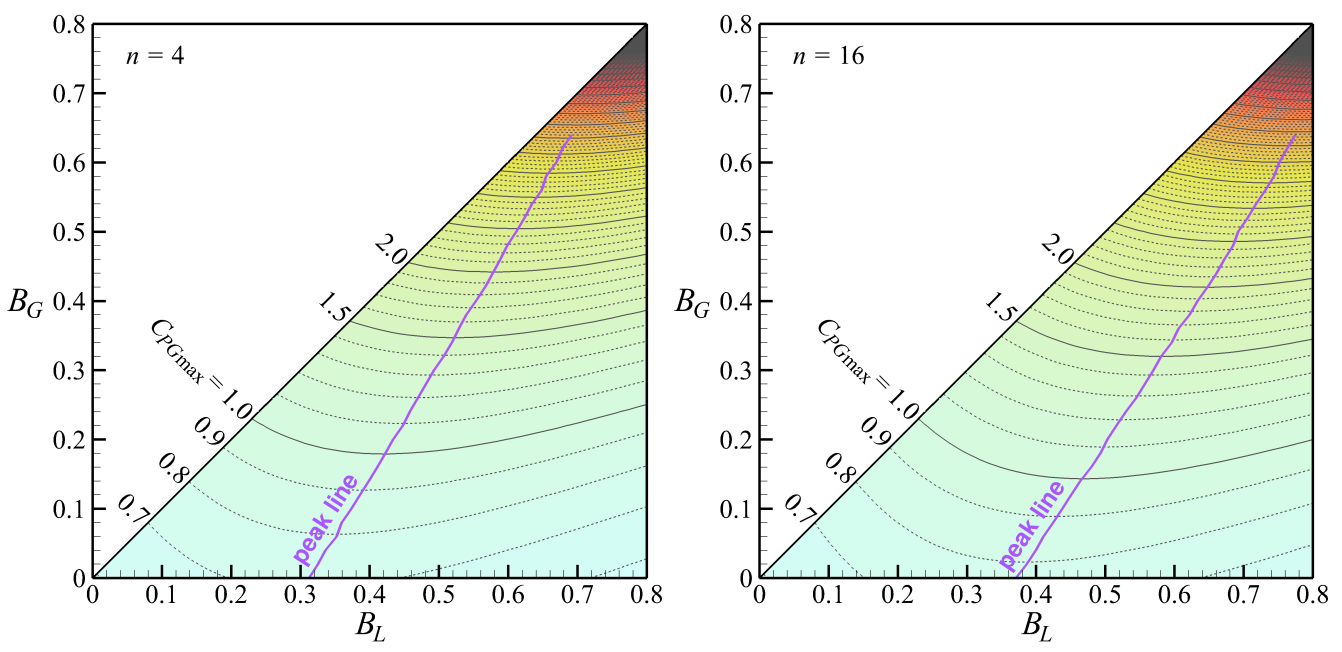

Figure 5. Combined effects of $B_{L}$ and $B_{G}$ on $C_{P G \max }$ for $n=4$ and 16 .

example, $C_{P G \max }$ increases from 1.65 for the 'full fence' configuration $\left(B_{L}=0.4\right)$ up to 1.75 (for $n=4$ ) and 1.88 (for $n=16$ ) as $B_{L}$ is optimised for each case.

It should be reminded that when $B_{L}=B_{G}$ the present model returns to the local (or 'full fence') power extraction model of Garrett \& Cummins (2007), where the number of devices does not affect the prediction of fence efficiency (as the array-scale flow expansion does not take place). The effect of the number of devices becomes more significant as $B_{L}$ becomes larger than $B_{G}$.

\section{Three-dimensional RANS computations}

So far we have investigated fundamental performance characteristics of relatively short and long partial tidal fences using the new analytical model. In this section, we present three-dimensional RANS computations of confined channel flow past various numbers (up to 40) of actuator disks to further investigate the characteristics of tidal fences as 


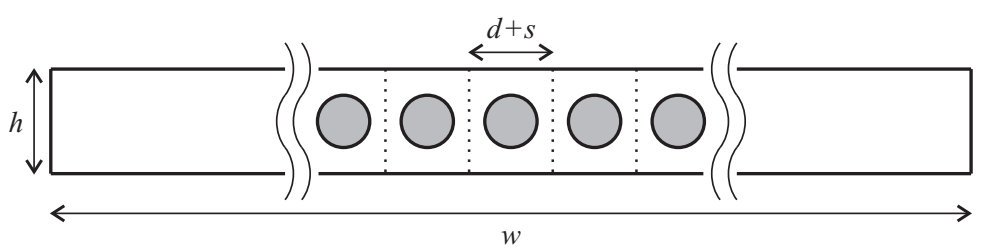

FIGURE 6. Schematic of the channel cross-section for RANS actuator disk computations.

$\begin{array}{cccccc}s / d & h / d & w / n d & n & B_{L} & B_{G} \\ 0.1 & 2 & 10 & 2,4,8 & 0.357 & 0.039 \\ 0.25 & 2 & 10 & 2,4,8,16,24,32,40 & 0.314 & 0.039 \\ 0.5 & 2 & 10 & 2,4,8 & 0.262 & 0.039 \\ 1 & 2 & 10 & 2,4,8 & 0.196 & 0.039\end{array}$

TABLE 1. Summary of flow configuration for RANS actuator disk computations

well as to examine the validity of the analytical model. The present RANS actuator disk methodology has been previously used by Nishino \& Willden (2012a) to investigate the effect of three-dimensional channel blockage on single actuator disk performance, which agrees very well with the analytical (one-dimensional) model prediction of Garrett \& Cummins (2007) when turbulent mixing in the near wake region (modelled in the RANS computations) is not significant. Details of the computations are described below, followed by results compared to the analytical partial fence model.

\subsection{Flow configuration}

Figure 6 shows a schematic of the cross-section of the channel simulated. We consider $n$ actuator disks of diameter $d$ arrayed regularly but only around the centre of the channel cross-section of height $h$ and width $w$. The spacing between each disk is $s$; therefore the local blockage $B_{L}=\pi d^{2} / 4 h(d+s)$ and global blockage $B_{G}=n \pi d^{2} / 4 h w$. Cartesian coordinates $(x, y, z)$ are employed, representing the streamwise, vertical and spanwise directions, respectively.

Table 1 summarises the flow configuration. In this study the channel height $h$ is fixed at $2 d$ whereas the channel width $w$ is set at $10 n d$ (i.e. the channel width is proportional to the number of disks) so that the global blockage is fixed. The intra-disk spacing $s$ is varied between $0.1 d$ and $1 d$; thus the local blockage is varied between 0.357 to 0.196 .

For the sake of expedience, here we suppose that the disk diameter $d=20 \mathrm{~m}$ and the channel inlet velocity $u=2 \mathrm{~m} / \mathrm{s}$, resulting in the Reynolds number $R e=\rho u d / \mu=4 \times 10^{7}$ (where $\rho=1000 \mathrm{~kg} / \mathrm{m}^{3}$ and $\mu=0.001 \mathrm{~kg} / \mathrm{m} \cdot \mathrm{s}$ are the density and viscosity of water). It should be noted, however, that the flow simulated upstream of the disks is nearly inviscid (as the channel inlet turbulence level is set very low in the present RANS computations, as will be described later) whereas the rate of mixing simulated downstream of the disks is predominantly determined by the turbulence model (as will be discussed later).

\subsection{Computational methods}

The governing equations to be solved are the three-dimensional incompressible 'steady' RANS equations (together with the continuity equation). The Reynolds stress terms are modelled using the $k-\epsilon$ eddy viscosity model of Launder \& Spalding (1974). Similarly to Nishino \& Willden (2012a), each power-extracting device (or turbine) is modelled as 
a stationary permeable disk; all disks are placed perpendicular to the $x$-axis and are located at $x=0$. The effect of each disk on the (Reynolds-averaged or 'mean') flow is considered as a loss of momentum in the streamwise direction at the disk plane. The change in momentum flux (per unit area and per unit density; to be added as 'source' of momentum to the RANS equation for the streamwise direction at the disk plane) is locally calculated as

$$
S_{U}=K\left(\frac{1}{2} U_{d}^{2}\right)
$$

where $U_{d}(y, z)$ is the local (rather than disk-averaged) streamwise velocity at the disk plane and $K$ is the so-called momentum loss factor (the parameter that determines the 'operating condition' of the devices). In this study $K$ is assumed to be uniform across the surface of all $n$ disks. Hence the thrust on each disk is calculated as

$$
T_{d(i)}=\int_{\operatorname{disk}(i)} \rho S_{U} \mathrm{~d} A_{d}=\frac{1}{2} \rho K \int_{\operatorname{disk}(i)} U_{d}^{2} \mathrm{~d} A_{d},
$$

where the integration is taken over the area of each disk, $A_{d}$, and $i$ denotes the $i$-th disk in the array of $n$ disks. Hence the total thrust on the $n$ disks is calculated as

$$
\sum_{i=1}^{n} T_{d(i)}=\frac{1}{2} \rho K \sum_{i=1}^{n} A_{d}\left\langle U_{d}^{2}\right\rangle_{i}=\frac{1}{2} \rho K n A_{d}\left\langle U_{d}^{2}\right\rangle
$$

where $\langle\phi\rangle_{i}$ indicates the face average of a variable $\phi$ over the $i$-th disk and $\langle\phi\rangle$ the face average of $\phi$ over all $n$ disks. Thus the (n-disk-averaged) global thrust coefficient, $\left\langle C_{T G}\right\rangle$, is calculated as

$$
\left\langle C_{T G}\right\rangle=\frac{\sum_{i=1}^{n} T_{d(i)}}{\frac{1}{2} \rho u^{2} n A_{d}}=K \frac{\left\langle U_{d}^{2}\right\rangle}{u^{2}} .
$$

Similarly, the power removed from the mean flow by the $i$-th disk at the disk plane is calculated as

$$
P_{d(i)}=\int_{\operatorname{disk}(i)} \rho S_{U} U_{d} \mathrm{~d} A_{d}=\frac{1}{2} \rho K \int_{\operatorname{disk}(i)} U_{d}^{3} \mathrm{~d} A_{d},
$$

hence the (n-disk-averaged) global power coefficient, $\left\langle C_{P G}\right\rangle$, is calculated as

$$
\left\langle C_{P G}\right\rangle=\frac{\sum_{i=1}^{n} P_{d(i)}}{\frac{1}{2} \rho u^{3} n A_{d}}=K \frac{\left\langle U_{d}^{3}\right\rangle}{u^{3}} .
$$

Also, we define the ( $n$-disk-averaged) global axial induction factor, $\left\langle a_{G}\right\rangle$, as

$$
\left\langle a_{G}\right\rangle=1-\frac{\left\langle U_{d}\right\rangle}{u} .
$$

As discussed in detail by Nishino \& Willden (2012a), this actuator disk method does not account for the effect of turbulence (or time-dependent fluid motion) generated by the device itself (i.e. wake turbulence is generated solely by the mean wake shear); hence the wake mixing simulated downstream of the disks tends to be rather weak (compared to, for example, that of common horizontal-axis turbines). Since the analytical tidal fence model to be compared with the present RANS computations is concerned with the hydrodynamic limit of power extraction (by some ideal devices that do not lose power to heat, turbulence etc., cf. Section 2.6), it might seem to be reasonable to ignore the effect 
of turbulence generated by the devices in the RANS computations as well. However, the analytical tidal fence model assumes that the device-scale wake mixing is completed in the array near-wake region (i.e. station $5 \mathrm{~L}$ is somewhere upstream of station $4 \mathrm{~A}$ ), which means that the analytical tidal fence model supposes some form of practical (strong) device-scale wake mixing. Note that $n P_{D}$ in the analytical model represents the power removed from the mean flow at the device place, which is indeed the hydrodynamic limit of power extraction, but the analytical model never knows whether the removed power is actually extracted as useful power or lost to generate heat, turbulence etc.; hence it is natural to consider that the analytical tidal fence model does implicitly account for the generation of turbulence at the devices (or more precisely, time-dependent fluid motion that enhances the mixing of bypass and core flows only in the device far-wake region, because the present analytical model does not account for the device near-wake mixing between stations 3 and $4 \mathrm{~L}$; for example, helical tip-vortices from horizontal-axis turbines might be close to this type of motion as they seem to significantly enhance the mixing only after the breakdown of their structures (Nishino \& Willden 2012c)).

A possible approach to account for the generation of turbulence at the devices within the framework of RANS actuator disk computations has been proposed by Nishino \& Willden (2012a), who modelled the additional turbulence (called blade-induced turbulence or BIT) based on two physical parameters: (i) ratio of the power converted to BIT to the power removed from the mean flow, and (ii) representative length scale for BIT. A difficulty here, however, is that the effect of modelled BIT on the device wake mixing is still rather hypothetical since the wake structure of actuator disks is different from that of actual devices; see Nishino \& Willden $(2012 a)$ for further discussion on the BIT approach. In order to avoid unnecessary complication, in this study we do not employ the BIT approach but simply manipulate one of the empirical model constants in the $k-\epsilon$ model of Launder \& Spalding (1974) to simulate stronger and weaker wake mixing cases. Specifically, we use three different values for the model constant for the production term in the $\epsilon$-equation, $C_{\epsilon 1}$ (which is denoted by $C_{1}$ in the original paper by Launder and Spalding): $C_{\epsilon 1}=1.36,1.44$ and 1.52. Unless specified, $C_{\epsilon 1}=1.44$ (the 'standard' value proposed by Launder \& Spalding) is used in this paper. Note that the smaller $C_{\epsilon 1}$ results in stronger wake mixing whereas the larger $C_{\epsilon 1}$ results in weaker wake mixing; however, it should be remembered that these 'stronger' and 'weaker' cases are just relative to the nominal 'standard' case, which itself does not represent anything physically standard in terms of simulating wake mixing of unknown devices.

Computations were performed using a second-order finite volume method employing a procedure similar to Rhie \& Chow (1983). The SIMPLE algorithm (Patankar 1980) was used for pressure-velocity coupling. See Nishino \& Willden (2012a) for further details of the computational methods.

\subsection{Boundary conditions and computational grids}

Since the flow configuration to be investigated is symmetric in both vertical and spanwise directions (as shown earlier in figure 6), computations were performed only for a quarter of the channel cross-section with symmetry boundary conditions applied to $y=0$ and $z=0$ planes. Note that the centre of the $n$-disk array is located at $(x, y, z)=(0,0,0)$. Inviscid or 'slip' wall boundary conditions are applied to the vertical and spanwise ends ( $y=h / 2$ and $z=w / 2$ planes). Of importance here is that the computational domain must be sufficiently long in the streamwise $(x)$ direction so that neither inlet nor outlet boundary would alter the flow around the disks (this theoretically means that the region between stations $1 \mathrm{~A}$ and $4 \mathrm{~A}$ needs to be contained in the computational domain).

Our preliminary computations suggested that the effect of the computational domain 


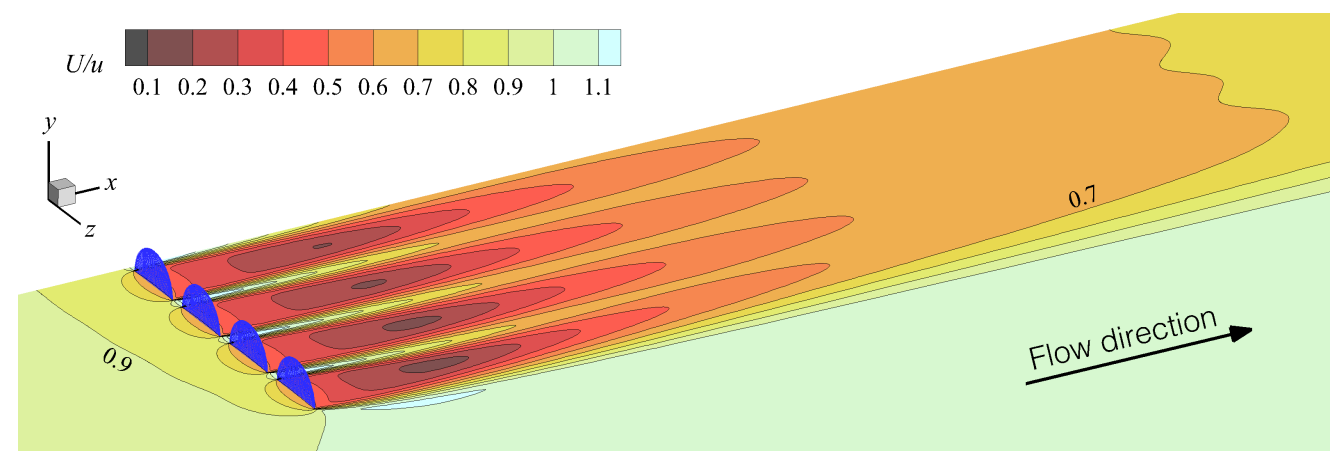

FiguRE 7. Example of streamwise velocity contours on the hub-height $(y=0)$ plane, together with computational mesh for actuator disks $\left(n=8, s / d=0.25,\left\langle a_{G}\right\rangle=0.4,\left\langle C_{P G}\right\rangle=0.742\right)$.

length was negligible when the distances from the devices to the inlet and outlet of the domain were both longer than the domain width (i.e. $w / 2$ ). Hence in this study we use the following two different types of computational grids: Grid A has a shorter domain $(-50 \leqslant x / d \leqslant 50)$ and finer grid density, used for the cases with $n \leqslant 8$, and Grid B has a longer domain $(-400 \leqslant x / d \leqslant 400)$ and coarser grid density, used for the cases with $n \geqslant 8$. Both grids were tested for $n=8$ cases and yielded practically identical results (differences in $\left\langle C_{P G}\right\rangle$ were about $0.5 \%$ at the maximum). The finer grid density (as well as the grid topology) employed for Grid A is comparable to that for 'medium resolution' previously used by Nishino \& Willden $(2012 a)$ for the single disk study.

For the inlet boundary conditions, uniform streamwise velocity $u(2 \mathrm{~m} / \mathrm{s})$ is prescribed together with very low values of turbulence model quantities $\left(k=6 \times 10^{-6} \mathrm{~m}^{2} / \mathrm{s}^{2}\right.$ and $\epsilon=1.2 \times 10^{-9} \mathrm{~m}^{2} / \mathrm{s}^{3}$; note that the results are insensitive to the inlet values of turbulence quantities as long as they are small enough). For the outlet boundary, zero streamwisegradient conditions are applied.

\subsection{Results}

Figure 7 shows an example of streamwise velocity contours on the 'hub-height' $(y=0)$ plane (for $n=8, s / d=0.25$ ) plotted together with computational mesh for the disks; note that only 4 half-disks are shown here due to the symmetry at $y=0$ and $z=0$. The global induction factor $\left\langle a_{G}\right\rangle=0.4$, which is close to the 'optimal' operating condition to maximise $\left\langle C_{P G}\right\rangle$ for this particular case. The contour lines upstream of the disks clearly indicate the two different scales of flow expansion, i.e., array-scale core flow deceleration occurs first upstream of the entire array and then device-scale core flow deceleration just upstream of each disk. It can also be seen that the device-scale wake mixing takes place much faster than the array-scale wake mixing.

Figure 8 compares hub-height streamwise velocity profiles at $x / d=-0.5,0,1$ and 5 for three different values of turbulence model coefficient $C_{\epsilon 1}(1.36,1.44,1.52)$. Although there are several possible ways to compare the flow fields, here we fix the global induction factor $\left\langle a_{G}\right\rangle$ at 0.4 for all three cases (note that $\left\langle a_{G}\right\rangle$ cannot be directly specified as an input but be obtained as a result of each computation; therefore several different values of the momentum loss factor $K$ were tested for each case to match $\left\langle a_{G}\right\rangle$ ). As can be seen from the figure, the value of $C_{\epsilon 1}$ does not affect the flow upstream of the disks (since the flow is practically inviscid there) but alters the rate of mixing downstream of the disks. The overall characteristics of the flow field, however, are still similar between these three cases; the device-scale mixing takes place much faster than the array-scale mixing. It can 

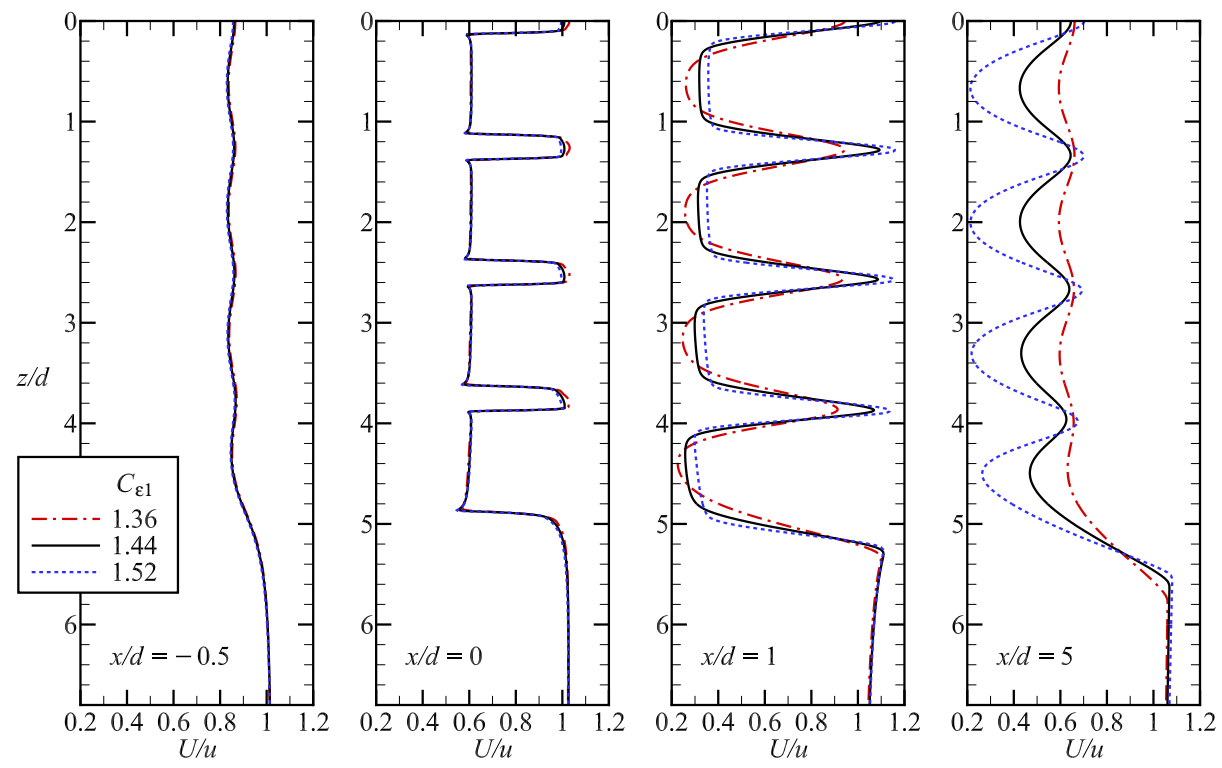

FiguRE 8. Effects of turbulence model constant $C_{\epsilon 1}$ on hub-height streamwise velocity profiles at $x / d=-0.5,0,1$ and 5 (for $n=8, s=0.25,\left\langle a_{G}\right\rangle=0.4$ ).
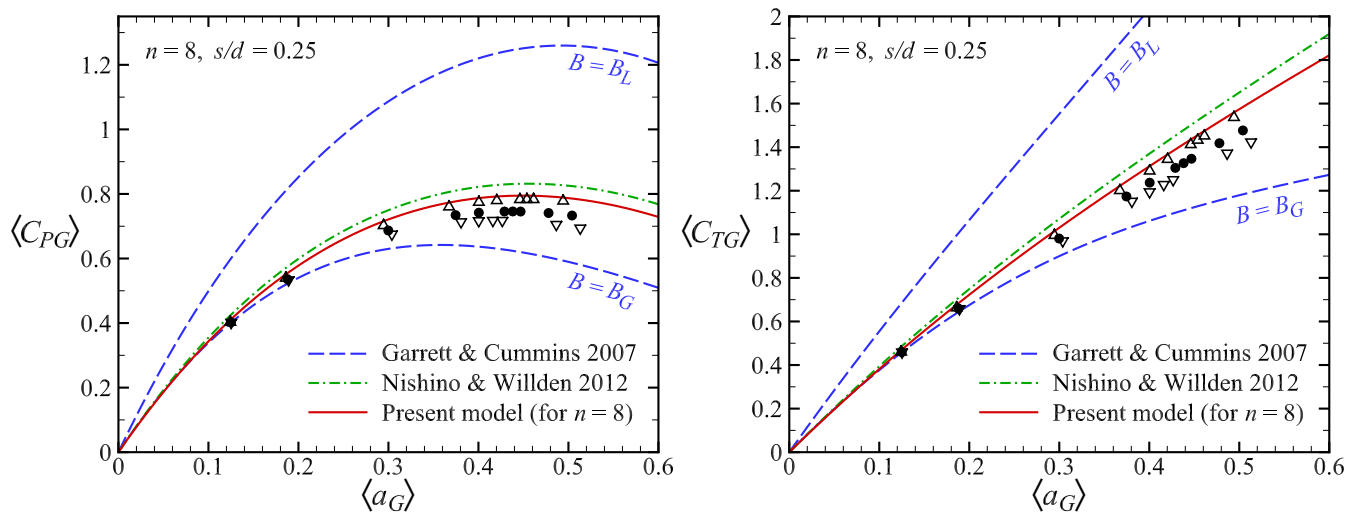

FIGURE 9. Comparisons of global power and thrust coefficients between RANS computations $\left(\triangle: C_{\epsilon 1}=1.36 ; \bullet: C_{\epsilon 1}=1.44 ; \nabla: C_{\epsilon 1}=1.52\right)$ and analytical models; for $n=8, s / d=0.25$.

also be seen that the streamwise velocity is nearly uniform across all disks (at $x / d=0$ ); the velocity is slightly lower at the outermost disks as the flow is oblique near the ends of the disk array.

Now we compare the prediction of fence performance between these computations and the analytical tidal fence model presented in Section 2. Figure 9 shows global power and thrust coefficients obtained from the RANS computations (using the three different $C_{\epsilon 1}$ values) and from the analytical model (for $n=8$ and $s / d=0.25$; hence $B_{L}=0.314$ and $B_{G}=0.039$ as listed in table 1). Also plotted here for comparison are the predictions obtained from the local (i.e. single-scale) model of Garrett \& Cummins (2007) and the original partial tidal fence model of Nishino \& Willden (2012b). Note that for the local model of Garrett \& Cummins (2007) there are two possible ways to define the blockage 


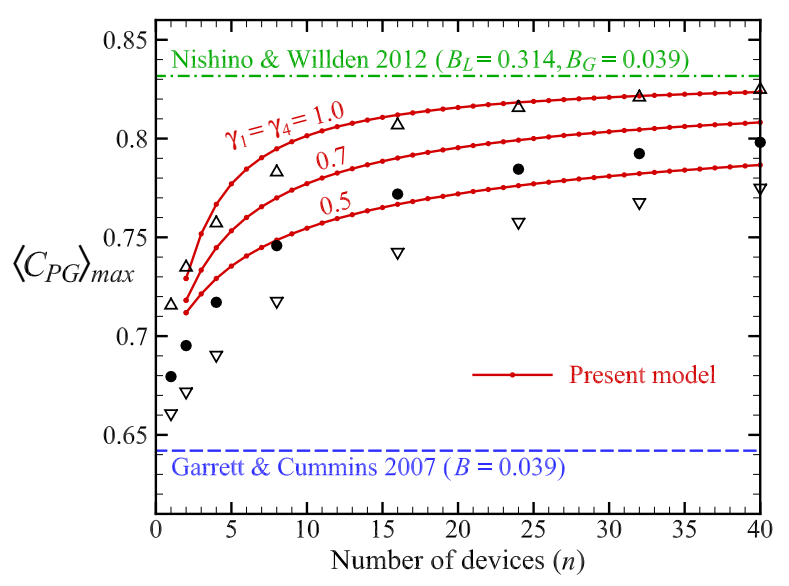

FIGURE 10. Effect of the number of devices on the maximal global power coefficient: obtained from RANS computations $\left(\triangle: C_{\epsilon 1}=1.36 ; \bullet: C_{\epsilon 1}=1.44 ; \nabla: C_{\epsilon 1}=1.52\right)$ and analytical models; for $s / d=0.25$.

$B$ for partial tidal fences: using the local blockage $\left(B=B_{L}\right)$ or using the global blockage $\left(B=B_{G}\right)$, both of which are plotted in the figure. As can be seen from the figure, RANS computations predict that both $\left\langle C_{P G}\right\rangle$ and $\left\langle C_{T G}\right\rangle$ gently increase as the mixing behind the disks is enhanced (by reducing $C_{\epsilon 1}$ ). The present analytical model agrees well with the RANS computations, especially those for the stronger mixing case $\left(C_{\epsilon 1}=1.36\right)$. The original partial fence model (Nishino \& Willden $2012 b$ ) slightly overpredicts both $\left\langle C_{P G}\right\rangle$ and $\left\langle C_{T G}\right\rangle$ but still agrees fairly well with the stronger mixing case.

Figure 10 compares the effect of the number of devices on the maximal global power coefficient (again for $s / d=0.25$; hence $B_{L}=0.314$ and $B_{G}=0.039$ ). Here the results of the present analytical model are plotted for three different values of $\gamma_{1}$ and $\gamma_{4}$ (the scaling exponents used in (2.3) and (2.4) to specify the dependence of array-scale flow expansion factors $\lambda_{1}$ and $\lambda_{4}$ on the number of devices $n$ ). It should be noted that $n$ is involved in the present analytical model only through the determination of $\lambda_{1}$ and $\lambda_{4}$ in (2.3) and (2.4); therefore the $\left\langle C_{P G}\right\rangle_{\max }$ versus $n$ curve is simply stretched toward larger $n$ as the scaling exponents $\gamma_{1}$ and $\gamma_{4}$ are reduced (for example, the prediction for $n=4$ and $\gamma_{1}=\gamma_{4}=1$ is identical to that for $n=16$ and $\left.\gamma_{1}=\gamma_{4}=0.5\right)$. As can be seen from the figure, the present analytical model using the 'default' scaling exponents $\left(\gamma_{1}=\gamma_{4}=1\right)$ agrees well with the RANS computations for the stronger mixing case $\left(C_{\epsilon 1}=1.36\right)$, although it seems that the agreement in the shape of the curve slightly improves if $\gamma_{1}$ and $\gamma_{4}$ are reduced to about 0.7 for this particular flow configuration; a possible reason for this will be discussed later in Section 4 .

Finally, figure 11 compares the effect of the intra-device spacing $s / d$ on the maximal global power coefficient (for $n=2,4$ and 8 ). Note that RANS results are plotted only for the stronger mixing case $\left(C_{\epsilon 1}=1.36\right)$ for comparison with the analytical model. Overall, RANS results agree fairly well with the analytical model; both indicate the trends that (i) the effect of the number of devices becomes more significant as the intra-device spacing decreases and (ii) the optimal spacing increases (i.e. optimal local blockage decreases) as the number of devices is reduced. Although not presented here, larger $C_{\epsilon 1}$ cases have also shown the same trends. It should be noted, however, that the present RANS results have shown a somewhat weaker dependence of the maximal power coefficient on $s / d$. For $n=4$ and 8 , for example, the analytical model predicts the maximal power coefficient 


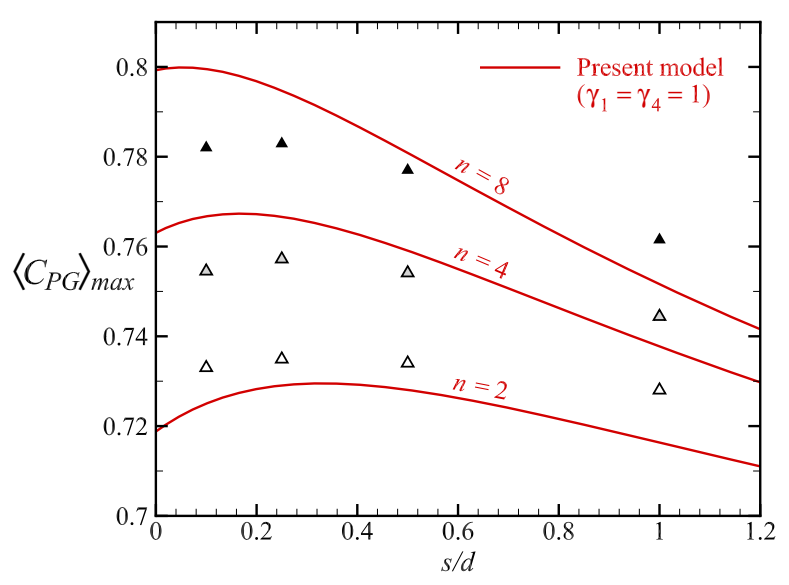

FiguRE 11. Effect of the intra-device spacing $s / d$ on the maximal global power coefficient: obtained from RANS computations with $C_{\epsilon 1}=1.36$ (symbols: black for $n=8$, grey for $n=4$ and white for $n=2$ ) and analytical model (lines).

slightly higher at smaller $s / d$ but lower at larger $s / d$ compared to the RANS results; this suggests that the analytical results would not perfectly match the RANS results by simply increasing or decreasing the scaling exponents $\gamma_{1}$ and $\gamma_{4}$. The results could match better if the scaling exponents are given as a function of $s / d$ or $B_{L}$; however, it should be remembered that there are several reasons why the analytical model does not (and should not) perfectly match the present RANS results, as will be discussed below.

\section{Discussion}

In this study we have compared a new two-scale (but still essentially one-dimensional) analytical model and three-dimensional RANS computations of partial tidal fence flows. On the whole, the new model agreed well with the RANS computations, suggesting that the two-scale dynamics described in the new analytical model predominantly determined the fence performance in the RANS computations as well. Most importantly, the model and computations have both demonstrated the trend that, for a given global blockage, the limit of power extraction (per device) by a partial tidal fence increases as the number of devices is increased. This is of importance when a certain number of devices are going to be installed across a given channel cross-section; for example, if we are going to install 8 devices in total, a single spanwise array of 8 devices (with its optimal local blockage) is expected to have a higher limit of power extraction than two spanwisely-separated spanwise arrays of 4 devices (with their optimal local blockage, which should be slightly smaller than that for an array of 8 devices).

It should be reminded that this kind of one-dimensional analytical model has a significant advantage in terms of computational cost compared to three-dimensional RANS computations, especially when optimising the configuration and operating conditions of a partial tidal fence at the same time. For example, the power coefficient contours shown in figure 3 (plotted against the local blockage and the wake mixing loss factor) are very useful for such an optimisation and can be easily obtained from an analytical model. To obtain these contours from RANS computations, we would need to consider a number of cases (for various combinations of array configuration and operating conditions), each of which requires a decent amount of computational resources. 


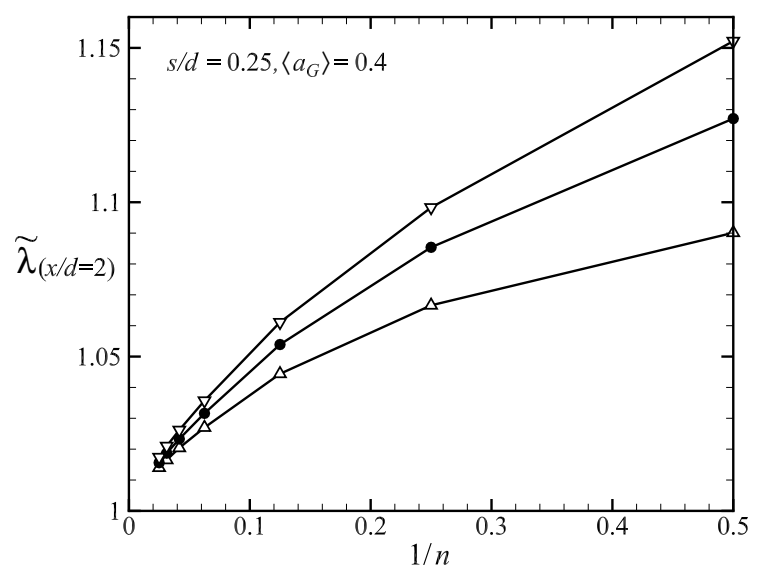

FigURE 12. Effect of the number of devices on the horizontal flow expansion factor at $x / d=2$ : obtained from RANS computations $\left(\triangle: C_{\epsilon 1}=1.36 ; \bullet: C_{\epsilon 1}=1.44 ; \nabla: C_{\epsilon 1}=1.52\right)$ for $s / d=0.25$ and $\left\langle a_{G}\right\rangle=0.4$.

The new analytical model and RANS computations, however, still showed some quantitative discrepancies between them. To examine the causes of discrepancies and also to better understand the fence flow mechanisms, here we discuss the following three issues: (i) modelling of array-scale flow expansion factors; (ii) effect of device near-wake mixing; and (iii) effect of device far-wake mixing.

The modelling of array-scale flow expansion factors $\lambda_{1}$ and $\lambda_{4}$ is an essential (and the only adjustable) part of the new analytical model. In this study we modelled $\lambda_{1}$ and $\lambda_{4}$ as in (2.3) and (2.4) and used $\gamma_{1}=\gamma_{4}=1$ as the 'default' scaling exponents; this means that the ratio of the array-scale stream-tube cross-sectional area at station $1 \mathrm{~L}$ to that at station 1A linearly decreases down to 1 (and similarly the ratio of the area at station $4 \mathrm{~L}$ to that at station $4 \mathrm{~A}$ linearly increases up to 1 ) as $1 / n$ increases from 0 to 1 . In reality, however, the dependence of $\lambda_{1}$ and $\lambda_{4}$ on $1 / n$ should be nonlinear mainly because the expansion of the array-scale stream-tube is nonlinear. Since its expansion rate is higher around the array than around stations $1 \mathrm{~A}$ and $4 \mathrm{~A}$, it seems reasonable to argue that the dependence of $\lambda_{1}$ and $\lambda_{4}$ on $1 / n$ should be more significant when $1 / n$ is small than when $1 / n$ is large (and hence the scaling exponents $\gamma_{1}$ and $\gamma_{4}$ should be less than 1 ). This argument can be supported by the results of RANS computations as shown in figure 12 . Since it is not straightforward to obtain $\lambda_{1}$ or $\lambda_{4}$ from the simulated three-dimensional flow data, here we plot the values of horizontal (two-dimensional) flow expansion factor $\tilde{\lambda}$ at $x / d=2$ (where the local pressure appears to approximately equilibrate) obtained from the following procedure: (i) extract the hub-height $(y=0)$ plane from the simulated flow data; (ii) calculate the two-dimensional stream-tube passing through the edge of 'fence area' (i.e. $x=0, z=n(d+s) / 2$ ); and (iii) calculate $\widetilde{\lambda}_{(x / d=2)}$ as the ratio of the width of this two-dimensional stream-tube at $x / d=2$ to that at $x / d=0$. Note that these values of $\widetilde{\lambda}$ are not directly comparable to $\lambda_{4}$ used in the analytical model; nevertheless, they show the nonlinear dependence of array-scale flow expansion factors on $1 / n$. It should also be noted that $\lambda_{1}$ and $\lambda_{4}$ are, in reality, expected to depend not only on the number of devices but also on the local and global blockages (and presumably the aspect ratios, rather than blockages, of local and channel flow passages as well) since they would affect the expansion rates of device- and array-scale stream-tubes. 
Although the modelling of array-scale flow expansion factors $\lambda_{1}$ and $\lambda_{4}$ is the source of uncertainty in the present analytical model, this is not the only cause of discrepancies between the analytical model and RANS computations. In other words, the analytical model would not perfectly agree with the RANS results even if the array-scale flow expansion factors were accurate. Two other major causes of discrepancies are: (i) device near-wake mixing (between stations 3 and $4 \mathrm{~L}$ ), which is neglected in the analytical model but not negligible in the RANS computations, and (ii) device far-wake mixing, which is assumed to be completed in the array near-wake region (upstream of station $4 \mathrm{~A}$ ) in the analytical model but this assumption is not fully satisfied in the RANS computations if the number of devices is small and/or the modelled turbulent wake mixing is weak. As concerns the effect of device near-wake mixing, Nishino \& Willden (2012a) have shown for single actuator disk cases that the maximal power removed at the disk increases as the mixing behind the disk is enhanced (and hence RANS actuator disk computations tend to somewhat overpredict the power coefficient compared to the analytical model of Garrett \& Cummins (2007); see Nishino \& Willden (2013) for further discussion on this issue). A similar effect can therefore be expected for the present tidal fence cases as well, i.e., the RANS computations should have a tendency of overpredicting the power coefficient (compared to the analytical tidal fence model) as the device near-wake mixing is enhanced. This means that the RANS computations with weaker wake mixing would agree better with the analytical tidal fence model than those with stronger wake mixing if the effect of device near-wake mixing was the only cause of discrepancies. On the other hand, however, the RANS computations with stronger wake mixing tend to satisfy the device far-wake mixing assumption (that the mixing is completed in the array near-wake region) better than those with weaker wake mixing. Thus, in terms of the effect of device far-wake mixing, computations with stronger wake mixing should agree better with the analytical tidal fence model than those with weaker wake mixing.

To consider the effect of device-scale wake mixing is important not only to explain the discrepancies between the present model and computations but also to explore how the performance of partial tidal fences can be improved in practice. Although not presented here, our preliminary computations without using any turbulence models (i.e. laminar flow comutations) showed that the maximal power coefficient $\left\langle C_{P G \max }\right\rangle$ for $n=8$ was insensitive to $s / d$ and was practically identical to that for $n=1$ (for the same global blockage of $B_{G}=0.039$ ), suggesting the importance of device-scale wake mixing on the fence performance. (In these laminar flow computations the disk wakes were observed to be stable, presumably due to the symmetry boundary conditions imposed at $y=0$ and $z=0$.) Analogously to the array-scale flow expansion factor $\lambda_{4}$ that essentially represents how much the array-scale stream-tube expands whilst the device-scale bypass and core flow pressure equilibrates, another influential factor to the fence performance would be that representing how much of the device-scale wake mixing takes place whilst the arrayscale bypass and core flow pressure equilibrates. For a relatively long fence, this factor would not be of interest since the device-scale wake mixing would always be completed in the array near-wake region (as assumed in the present analytical model). For a short fence, however, this factor would depend on device wake structures and therefore on the design of devices to be arrayed. In other words, the limiting performance of short partial tidal fences could be improved by somehow enhancing the device-scale wake mixing.

\section{Conclusions}

In this study we analytically and computationally investigated the characteristics of flow past a partial cross-stream array of (idealised) tidal turbines to better understand 
the mechanisms that determine the limiting performance of partial tidal fences. A twoscale analytical partial tidal fence model previously proposed by Nishino \& Willden $(2012 b)$ has been further extended by better accounting for the effect of array-scale flow expansion on device-scale dynamics, so that the new model is applicable to short partial fences (consisting of a small number of devices, where the two scales are not completely separated) as well as to long partial fences.

The new analytical model has explained general trends of the limiting performance of partial tidal fences. Most importantly the model has explained that, even for a given global blockage (rather than for a given channel cross-sectional area), the limit of power extraction (per device area) by a partial tidal fence increases as the number of devices forming the array increases. This is essentially because the local blockage effect is enhanced as the scale separation between the device- and array-scale flow events becomes more significant. It has also been shown that, as the number of devices in the array increases, the balance between the (beneficial) effect of local blockage and the (adverse) effect of flow reduction for the entire array is altered in such a way that the optimal local blockage increases. These trends are expected not only for low global blockage cases but also for high global blockage cases.

The new analytical model has also been compared with three-dimensional RANS actuator disk computations of partial tidal fence flows, where three different levels of wake mixing strength were simulated. On the whole, the new analytical model agrees well with the RANS computations, suggesting that the two-scale dynamics described in the new analytical model predominantly determines the partial fence performance in the RANS computations as well. The present analytical model and RANS computations, however, have still shown some quantitative discrepancies between them. These discrepancies are considered to be partly due to the effect of device-scale far-wake mixing, which has been assumed in the analytical model to be completed within the array near-wake region but in reality this assumption may not fully hold when the number of devices in the array is small. In addition to the array-scale flow expansion factors considered in the new analytical model, the rate of device-scale wake mixing (or more precisely, how much of the device-scale mixing takes place within the array near-wake region) could be another influential factor to the limiting performance of short partial tidal fences. This factor, however, depends on device wake structures and therefore on the type/design of devices to be arrayed.

Finally, it should be noted that this study did not consider any channel-scale dynamics, i.e. we assumed a constant channel mass flow in order to focus on fundamental partial tidal fence flow dynamics (that is independent of the channel-scale dynamics). In a practical tidal channel that is a part of a larger flow system, the channel mass flow should change depending on the configuration and operating conditions of tidal devices unless the drag caused by the devices is small enough not to change the balance of flow resistance inside and outside the entire channel. Also note that the effects of the channel's bed friction and vertical shear of the channel flow were neglected in this study; further investigations are required to understand these effects. Nevertheless, the physical insight into the characteristics of partial tidal fence flows obtained in this study should remain of fundamental importance.

The authors gratefully acknowledge the support of the Oxford Martin School, University of Oxford, who have funded this research.

\section{REFERENCES}


Corten, G. P. 2000 Heat generation by a wind turbine. In Proc. 14th IEA Symposium on the Aerodynamics of Wind Turbines. NREL, CO, USA.

Garrett, C. \& Cummins, P. 2005 The power potential of tidal currents in channels. Proc. R. Soc. A 461, 2563-2572.

Garrett, C. \& Cummins, P. 2007 The efficiency of a turbine in a tidal channel. J. Fluid Mech. 588, 243-251.

Houlsby, G. T., Draper, S. \& Oldfield, M. L. G. 2008 Application of linear momentum actuator disc theory to open channel flow. Tech. Rep.. OUEL 2296/08, Dept. Engineering Science, University of Oxford.

Launder, B. E. \& Spalding, D. B. 1974 The numerical computation of turbulent flows. Comput. Method. Appl. M. 3, 269-289.

Nishino, T. \& Willden, R. H. J. $2012 a$ Effects of 3-d channel blockage and turbulent wake mixing on the limit of power extraction by tidal turbines. Int. J. Heat Fluid Flow 37, $123-135$.

Nishino, T. \& Willden, R. H. J. $2012 b$ The efficiency of an array of tidal turbines partially blocking a wide channel. J. Fluid Mech. 708, 596-606.

Nishino, T. \& Willden, R. H. J. $2012 c$ Low-order modelling of blade-induced turbulence for rans actuator disk computations of wind and tidal turbines. In Proc. EUROMECH Colloquium 528: Wind Energy and the Impact of Turbulence on the Conversion Process. Oldenburg, Germany.

Nishino, T. \& Willden, R. H. J. 2013 The efficiency of tidal fences: a brief review and further discussion on the effect of wake mixing. In Proc. 32nd International Conference on Ocean, Offshore and Arctic Engineering (OMAE2013). Nantes, France.

PAtAnkar, S. V. 1980 Numerical heat transfer and fluid flow. Hemisphere Publishing Corporation.

Rhie, C. M. \& Chow, W. L. 1983 Numerical study of the turbulent flow past an airfoil with trailing edge separation. AIAA J. 21, 1525-1532.

Vennell, R. 2010 Tuning turbines in a tidal channel. J. Fluid Mech. 663, 253-267.

Vennell, R. 2011 Tuning tidal turbines in-concert to maximise farm efficiency. J. Fluid Mech. 671, 587-604.

Vennell, R. 2012 The energetics of large tidal turbine arrays. Renewable Energy 48, 210-219.

VenNell, R. 2013 Exceeding the betz limit with tidal turbines. Renewable Energy 55, 277-285.

Whelan, J. I., Graham, J. M. R. \& Peiró, J. 2009 A free-surface and blockage correction for tidal turbines. J. Fluid Mech. 624, 281-291. 